\title{
Carbon nanotubes as potential material for drug delivery-experiment and simulation
}

\author{
Artur P. Terzyk • Marek Wiśniewski • Katarzyna Dulska • Agnieszka Bielicka • \\ Piotr A. Gauden • Sylwester Furmaniak • Karolina Werengowska-Ciećwierz
}

Received: 24 October 2012 / Accepted: 28 November 2012 / Published online: 6 December 2012

(C) The Author(s) 2012. This article is published with open access at Springerlink.com

\begin{abstract}
We discuss the factors influencing the properties of new drug delivery system, composed of carbon nanotubes and analgesic antipyretic drug-paracetamol. Basing on experimental data it is shown, that by a simple manipulation with the heating time at the stage of system preparation, one can easily change the rate of the drug delivery. Moreover, this rate can be changed in a very wide range. Finally, using Molecular Dynamics simulation we also discuss the orientation and properties of drug molecules at different stages of the hot melt deposition process.
\end{abstract}

Keywords Drug delivery $\cdot$ Paracetamol $\cdot$ Nanotubes $\cdot$ Hot melt drug deposition

Carbon nanotubes become more and more important in construction of drug delivery vehicles (Pańczyk et al. 2011; Peretz and Regev 2012). It should be mentioned, however, that the problem of nanotubes toxicity issue still awaits a consensus, as demonstrated by inconclusive or contradictory studies. This problem should be resolved before the first practical applications of CNT-based medicine are approved (Peretz and Regev 2012).

Recently the first application of carbon nanotubes in so-called hot melt drug deposition process was proposed (Terzyk et al. 2012). It was proven using thermal analysis, DSC and desorption kinetics measurements (as well as molecular simulations) that paracetamol molecules are adsorbed between carbon nanotubes and form nanoaggregates.

\footnotetext{
A.P. Terzyk $(\varangle) \cdot$ M. Wiśniewski · K. Dulska · A. Bielicka · P.A. Gauden · S. Furmaniak - K. Werengowska-Ciećwierz Department of Chemistry, Physicochemistry of Carbon Materials Research Group, N. Copernicus University, Gagarin Street 7, 87-100 Toruń, Poland

e-mail: aterzyk@chem.uni.torun.pl
}

After heating and after evaporation of the solvent nanoaggregates are destroyed, and drug molecules are redistributed and adsorbed on the walls of carbon nanotubes. We also showed (for a single case) that the rate of desorption of the drug adsorbed in this way is remarkably slower than the rate of desorption of the drug adsorbed in the form of nanoaggregates (i.e. without heating).

In this way, by a simple thermal treatment of drug/nanotubes mixture, one can easily change the rate of desorption of a drug and to prepare the drug delivery system having well defined properties. Figure 1 shows selected parameters influencing the behavior of new drug delivery systems obtained using the hot-melt drug deposition process. As one can see on Fig. 1 among the most important factors influencing the properties of new carbon-based drug delivery system (for a chosen drug) we can mention: the temperature of deposition process, mixing conditions, tube properties and finally the conditions of drug delivery process ( $\mathrm{pH}, T$, etc.).

The major aim of this communication is to estimate, for one arbitrarily chosen system, how wide range of delivery rate can be achieved by changing the values of selected parameters mentioned in Fig. 1.

We choose carbon nanotubes called Baytubes C 150 HP (Bayer Material Science, Germany), characterized recently (Furmaniak et al. 2010; Wiśniewski et al. 2010). The BET surface area is equal to $198 \mathrm{~m}^{2} / \mathrm{g}$, and the distribution of external diameters shows a maximum at c.a. $15 \mathrm{~nm}$ (Furmaniak et al. 2010). Nanotubes were mixed with paracetamol (Merck, Germany) and we present the results for a fixed (i.e. equal to 0.4 ) drug to carbon ratio. This ratio means that per $1 \mathrm{~g}$ of carbon nanotubes we used $0.4 \mathrm{~g}$ of the drug. Both components were mixed using laboratory ball mill, next placed in the oven $(T=473 \mathrm{~K})$ and heated from 0.5 up to 3.0 hours. We used the following notation of studied samples: the name of material, the mass of drug (per 


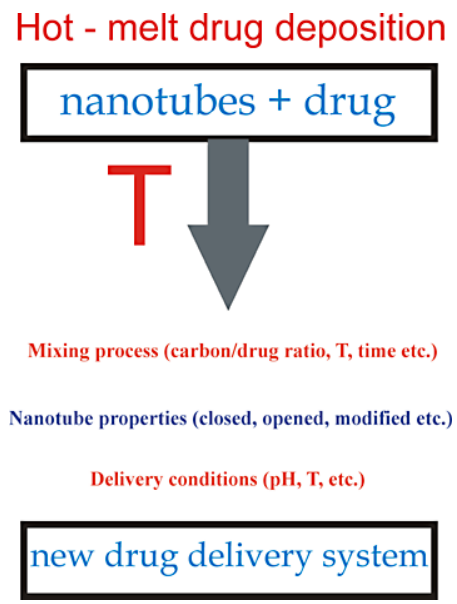

Fig. 1 Schematic representation of the hot melt drug deposition process and the major parameters changing the properties of obtained in this way drug delivery systems

$1 \mathrm{~g}$ of carbon material), and the time of hot melt deposition (in hours) process. Thus for example: B-0.4-1.0 denotes the sample of carbon Baytubes containing $0.4 \mathrm{~g}$ of paracetamol per $1 \mathrm{~g}$ carbon material and heated for 1.0 hour. After hotmelt deposition process the samples were cooled down to the room temperature.

Delivery measurement was performed in the special tight vessel containing magnetic rotor. The vessel was placed in a thermostat, filled with $100 \mathrm{~cm}^{3}$ of water and the measurement temperature was equal to $310 \pm 0.1 \mathrm{~K}$ (mass of adsorbent $25 \mathrm{mg}$ ). Paracetamol concentration was determined using the UV-vis spectrophotometer (Jasco V 660, Japan) at the wavelength $244.6 \mathrm{~nm}$. Each kinetic curve was measured three times and the data were averaged.

As one can observe from Fig. 2, for a constant drug to carbon ratio (equal 0.4) we observe drastic difference in drug delivery rate for different heating times applied during the hot-melt deposition process. The longer is the heating time, the slower desorption process is. We observe very wide range of desorbed drug amount, therefore one can conclude that by changing the heating time one can easily manipulate with the rate of drug delivery. This is very important especially in drug delivery systems because in some cases it is necessary to deliver the drug rapidly, and sometimes it is necessary to deliver it very slowly, even for few weeks.

To perform a full characterization of a studied drug delivery system we tried to predict the kinetic curve for a selected time of heating. To do this the data collected in Fig. 2 were described using different kinetic models. Among them we have chosen a model having relatively good fitting quality but simultaneously, the smallest as possible number of best fit parameters. In the case of data collected in Fig. 2 the Pseudo Second Order (PSO) model is in our opinion the best

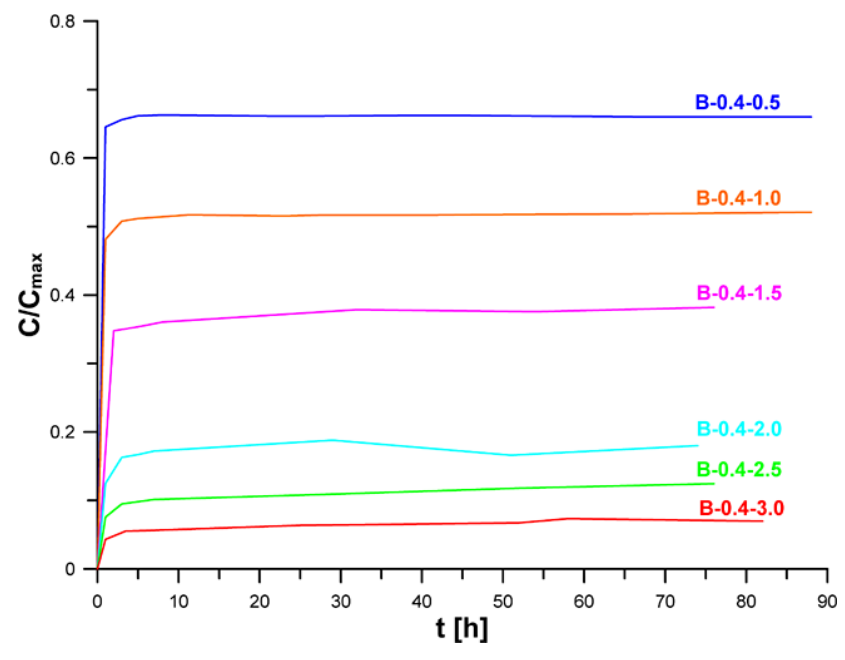

Fig. 2 Kinetics of paracetamol desorption from studied new drug delivery systems at $310 \mathrm{~K}$

choice (the DC value ranged from 0.96 to 0.99 ). The pseudo second order equation (Blanchard et al. 1984):

$\frac{d C^{*}}{d t}=k_{2}\left(C_{e}^{*}-C^{*}\right)^{2}$

was used in the form (Ho and McKay 1998):

$C^{*}=\frac{k_{2}\left(C_{e}^{*}\right)^{2} t}{1+k_{2} C_{e}^{*} t}$

where

$C^{*}=\frac{C}{C_{\max }}$

and $C$ is the actual concentration of released paracetamol in the solution, $C_{\max }$ is the theoretically calculated concentration corresponding to desorption of all paracetamol, $C_{e}^{*}$ corresponds to equilibrium condition, $k_{2}$ is the kinetic constant and $t$ is time. As it was pointed out by Azizian (2004) this equation describes experimental kinetics data if the initial concentration of a solute is not too high. Moreover, Ho et al. (1996) derived this equation assuming the presence of two types of adsorption sites on a surface.

In Fig. 3 we show the general correlations between the parameters of Eqs. (1) and (2) and the time of the hot melt drug deposition process. One can also conclude that the most drastic changes are observed during the first three hours of heating. However, the kinetic constant of Eq. (2) changes remarkably only for the first 1.5 hour of heating. Knowing the relations between both constants and the deposition time one can easily to plot the kinetic curve for all intermediate cases.

Finally to explain the behavior of kinetic curves collected in Fig. 2 we used Molecular Dynamics simulations (Gromacs package-see Terzyk et al. 2012 for details). Starting from 20 different configurations we performed MD simulations for $(13,0)$ nanotube and 75 paracetamol 

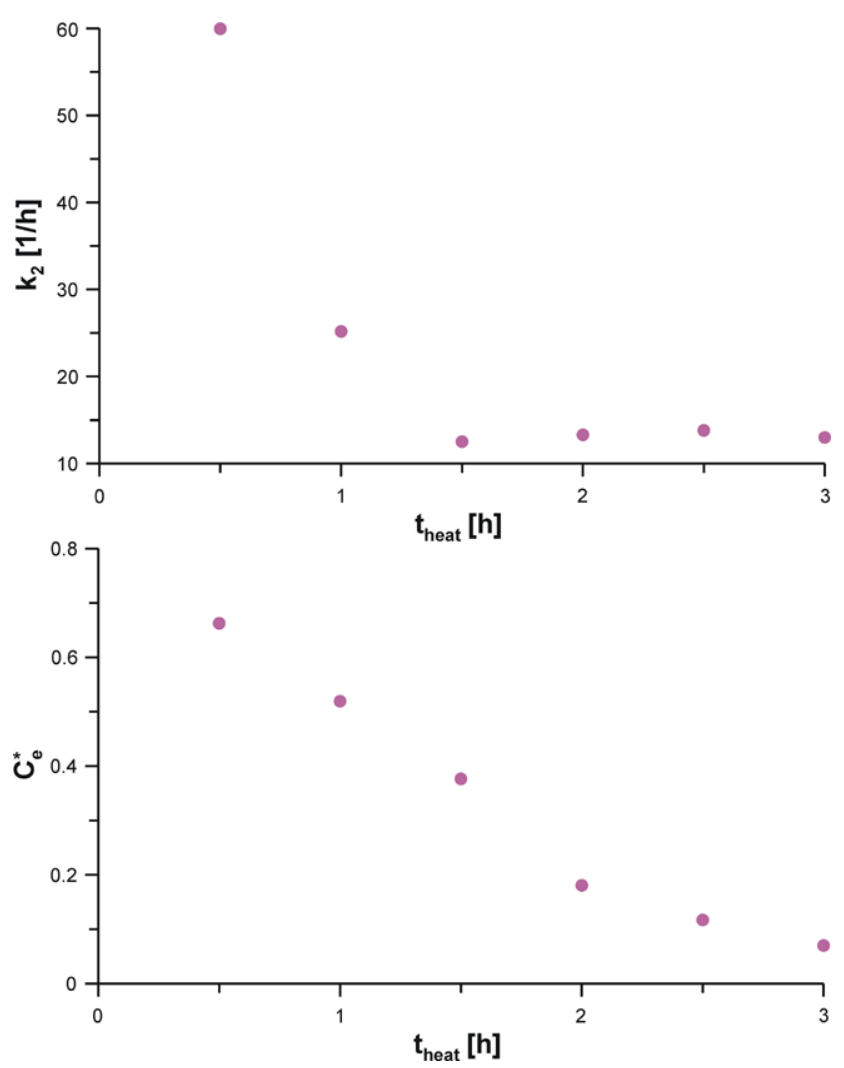

Fig. 3 The correlations obtained between the parameters of PSO model used to description of experimental delivery curves, and heating time during hot melt deposition process molecules $(T=473 \mathrm{~K})$. Next, we averaged the orientations of molecules from short time intervals shown in Fig. 4. In this way we have analogy to real experiment where using different times of heating of carbon/drug system the system is "frozen" on the path to the equilibrium.

In order to characterize the angular orientation of paracetamol molecules with respect of the nanotube surface, we calculated the angle $(\alpha)$ between the vector perpendicular to the benzene ring plane and the lengthening of the tube radius passing through the centre of this ring (Terzyk et al. 2009). Thus if $\alpha=0^{\circ}$ molecules are perfectly parallel to the tube. In contrast for $\alpha=90^{\circ}$ they have perpendicular orientation. We consider only the molecules at the vicinity of nanotubes assuming the distance between the centre of the ring and at least one carbon atom forming the tube was not larger than $0.8 \mathrm{~nm}$.

The data collected in Fig. 4 show that the maximum of the peak is located at the $\alpha=10^{\circ}$. Thus some molecules are almost parallel to the tube. However, with the rise in the time of the hot-melt deposition process (i.e. with the time of equilibration during MD simulation) the distribution becomes narrower i.e. the molecules tend to more parallel orientation. Since the orientation of molecules changes the rate of drug delivery, this simple simulation experiment shows that the differences observed in Fig. 3 can be caused by the orientation of adsorbed molecules. But the snapshots collected in Fig. 4 also show, that the number of molecules placed at larger distances from the tube decreases with the time of
Fig. 4 Total energy of the system and histogram of angular orientation of adsorbed molecules at different stages of the equilibration, together with selected snapshots
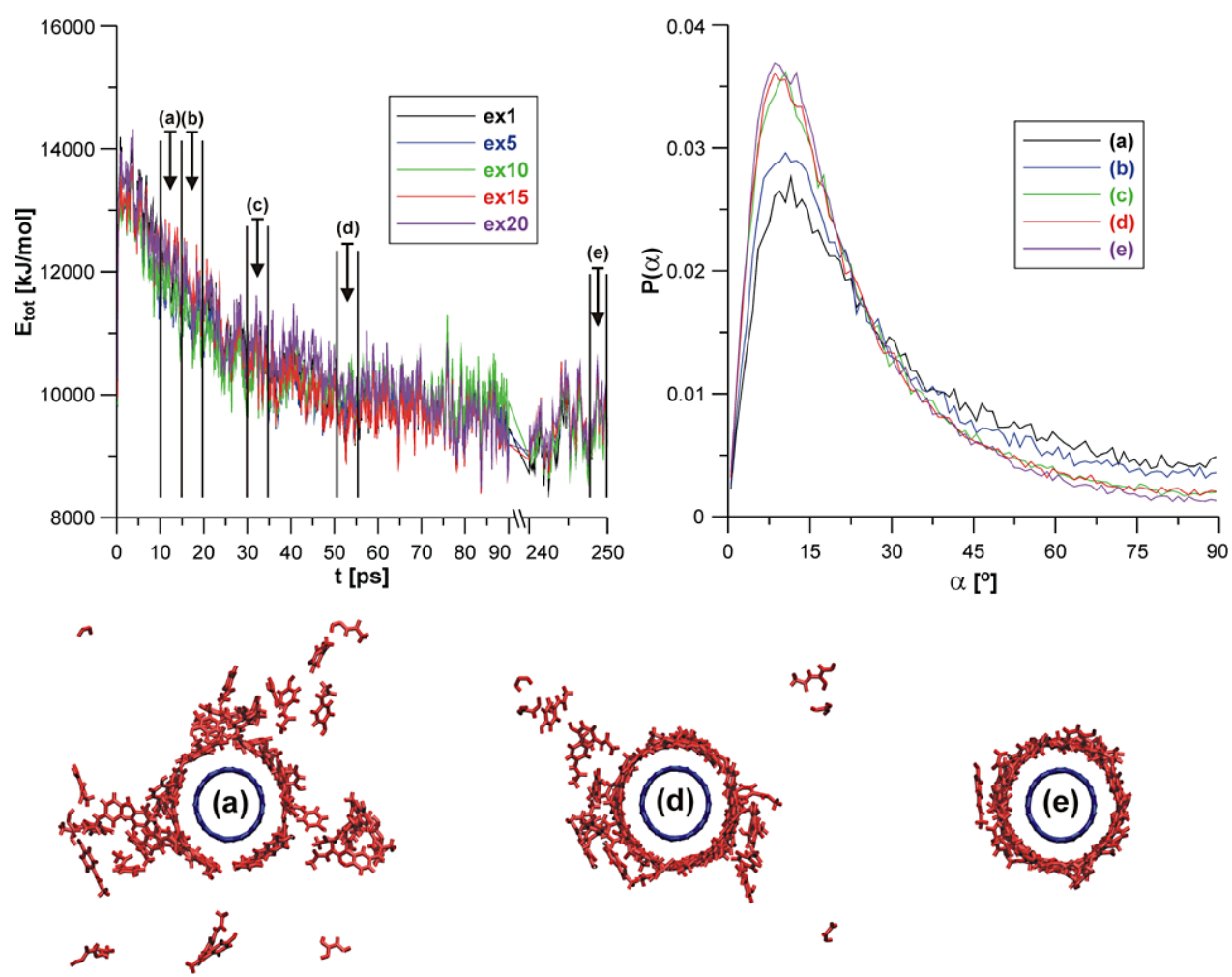
equilibration. And this is, in our opinion, the second reason explaining the behavior of kinetic curves collected in Fig. 2.

In summary we can state that proposed "hot melt deposition method" used for carbon nanotubes can be very promising for preparation of new drug delivery systems. It is simple, no time consuming, and what is the most importantleads to the drug delivery systems having very wide range of delivery times. Further studies on those systems are necessary and the results will be reported in future.

Acknowledgements A.P.T., M.W., P.A.G., and K.W.-Ć. gratefully acknowledge the financial support from NSC Grant DEC-2011/01/B/ ST5/01192. The authors acknowledge the use of the computer cluster at Poznań Supercomputing and Networking Centre (Poznań, Poland) and the Information and Communication Technology Centre of the Nicolaus Copernicus University (Toruń, Poland).

Open Access This article is distributed under the terms of the Creative Commons Attribution License which permits any use, distribution, and reproduction in any medium, provided the original author(s) and the source are credited.

\section{References}

Azizian, S.: Kinetic models of sorption: a theoretical analysis. J. Colloid Interface Sci. 276, 47-52 (2004)

Blanchard, G., Maunaye, M., Martin, G.: Removal of heavy metals from waters by means of natural zeolites. Water Res. 18, 15011507 (1984)
Furmaniak, S., Terzyk, A.P., Gauden, P.A., Harris, P.J.F., Wiśniewski, M., Kowalczyk, P.: Simple model of adsorption on external surface of carbon nanotubes-a new analytical approach basing on molecular simulation data. Adsorption 16, 197-213 (2010)

Ho, Y.S., Wase, D.A.J., Forster, C.F.: Kinetic studies of competitive heavy metal adsorption by sphagnum moss peat. Environ. Technol. 17, 71-77 (1996)

Ho, Y.S., McKay, G.: Sorption of dye from aqueous solution by peat. Chem. Eng. J. 70, 115-124 (1998)

Pańczyk, T., Camp, P.J., Pastorin, G., Warzocha, T.P.: Computational study of some aspects of chemical optimization of a functional magnetically triggered nanocontainer. J. Phys. Chem. C 115, 19074-19083 (2011)

Peretz, S., Regev, O.: Carbon nanotubes as nanocarriers in medicine. Curr. Opin. Colloid Interface Sci. 17, 360-368 (2012)

Terzyk, A.P., Gauden, P.A., Furmaniak, S., Wesołowski, R.P., Harris, P.J.F., Kowalczyk, P.: Adsorption from aqueous solutions on opened carbon nanotubes-organic compounds speed up delivery of water from inside. Phys. Chem. Chem. Phys. 11, 9341-9345 (2009)

Terzyk, A.P., Pacholczyk, A., Wiśniewski, M., Gauden, P.A.: Enhanced adsorption of paracetamol on closed carbon nanotubes by formation of nanoaggregates: Carbon nanotubes as potential materials in hot-melt drug deposition-experiment and simulation. J. Colloid Interface Sci. 376, 209-216 (2012)

Wiśniewski, M., Rychlicki, G., Arcimowicz, A.: Experimental and theoretical estimations of the polar force contributions to the heat of immersion of carbon nanotubes. Chem. Phys. Lett. 485, 331-334 (2010) 